\title{
Effect of static vs. dynamic imaging on particle transport in CT-based numerical models of human central airways
}

\author{
Shinjiro Miyawaki, ${ }^{1}$ Eric A. Hoffman, ${ }^{3,4,5}$ and Ching-Long Lin ${ }^{1,2, *}$ \\ ${ }^{1}$ IIHR-Hydroscience \& Engineering, \\ ${ }^{2}$ Department of Mechanical and Industrial Engineering, \\ ${ }^{3}$ Biomedical Engineering, ${ }^{4}$ Medicine and ${ }^{5}$ Radiology \\ The University of Iowa, Iowa City, Iowa 52242
}

*Corresponding author: Ching-Long Lin

Address: 3131 Seamans Center, Iowa City, Iowa 52242, U.S.A.,

Email: ching-long-lin@uiowa.edu, Tel: +1 3193355673 


\begin{abstract}
Advances in quantitative computed tomography (CT) has provided methods to assess the detailed structure of the pulmonary airways and parenchyma, providing the means of applying computational fluid dynamics-based modeling to better understand subject-specific differences in structure-to-function relationships. Most of the previous numerical studies, seeking to predict patterns of inhaled particle deposition, have considered airway geometry and regional ventilation derived from static images. Because geometric alterations of the airway and parenchyma associated with regional ventilation may greatly affect particle transport, we have sought to investigate the effect of rigid vs. deforming airways, linear vs. nonlinear airway deformations, and step-wise static vs. dynamic imaging on particle deposition with varying numbers of intermediate lung volume increments. Airway geometry and regional ventilation at different time points were defined by four-dimensional (space and time) dynamic or static CT images. Laminar, transitional, and turbulent air flows were reproduced with a three-dimensional eddyresolving computational fluid dynamics model. Finally, trajectories of particles were computed with the Lagrangian tracking algorithm. The results demonstrated that static-imaging-based models can contribute $7 \%$ uncertainty to overall particle distribution and deposition primarily due to regional flow rate (ventilation) differences as opposed to geometric alterations. The effect of rigid vs. deforming airways on serial distribution of particles over generations was significantly smaller than reported in a previous study that used the symmetric Weibel geometric model with smaller flow rate. Rigid vs. deforming airways were also shown to affect parallel particle distribution over lobes by $8 \%$ and the differences associated with use of static vs. dynamic imaging was $18 \%$. These differences demonstrate that estimates derived from static vs. dynamic imaging can significantly affect the assessment of particle distribution heterogeneity.
\end{abstract}


The effect of linear vs. nonlinear airway deformations was within the uncertainty due to mesh size.

Keywords: 4DCT, serial distribution, parallel distribution, computational fluid dynamics, image registration 


\section{Introduction}

Transport of particulate matter in the human lung is of great interest when seeking to understand the impact of environmental pollutants and cigarette smoke in the development of pulmonary pathology. In addition, efficacy of aerosolized pharmaceutical drugs are likely highly dependent on distribution patterns. For the former case, it is crucial to understand preferential locations of particle deposition based upon the given particle characteristics. In contrast, for the latter case, the motivation is to find the most effective particle characteristics to achieve the desired deposition pattern (Darquenne et al., 2016).

It would be ideal to measure transport of particles in vivo, e.g., using imaging techniques (Greenblatt et al., 2015). However, resolution of current imaging technologies (largely radionuclear-based) do not allow us to investigate particle transport in great detail. Another experimental approach is to use physical (in vitro) models (Grgic et al., 2004), but it is difficult to reproduce physiologically-realistic airway geometry and regional ventilation during breathing in a subject-specific manner. With advances in technology, computer (in silico) models have gained popularity in recent years (Choi et al, 2009; Ma and Lutchen, 2009). Although computer models include simplifications, the models have become more realistic with the aid of imaging techniques that allow, e.g., construction of subject-specific airway geometry and definition of regional ventilation during dynamic breathing (De Backer et al, 2010; Dubsky and Fouras, 2015; Yin et al., 2010). Given physiologically-realistic boundary conditions, three-dimensional (3-D) computational fluid dynamics (CFD) models can predict air flow and particle transport in central airways that are resolved by imaging with a reasonable accuracy (Choi et al., 2010; Miyawaki et al., 2012). 
According to Montaudon et al. (2009a, 2009b), the luminal areas of airways with generation numbers of four and higher in asthmatic subjects and smokers are significantly different from those of healthy subjects. Therefore, it is important to investigate particle transport not only in small airways, but also in central airways. A number of researchers have investigated particle transport in human central airways with CFD-based computer models. Two of the major subject-related factors that would influence the results of simulations are regional ventilation and airway geometry. Yin et al. (2010) prescribed regional ventilation by means of image registration and volume-filling technique. This method provides arguably the most physiologically-realistic boundary conditions to human lung models, although Ibrahim et al. (2015) took advantage of a vascular tree to reproduce more realistic sub-lobar ventilation of their rat lung model. Regarding airway geometry, most of the previous studies used rigid airways, while more recent studies used deforming airways based on static or dynamic images of the rat (Mead-Hunter et al., 2013; Ibrahim et al., 2016) and human lungs (Yin et al., 2013). Airway geometry based on static images may reflect very slow respiratory rates. However, dynamic images are required for the simulations of lungs breathing at more physiologically meaningful rates ( 4 sec/cycle).

Mead-Hunter et al. (2013) compared depositions of particles in a rigid and deforming Weibel-type geometric model, where deformation was defined by scaling relative to a fixed reference point. Yin et al. (2013) simulated air flow in a rigid, linearly deforming, and nonlinearly deforming imaging-based models of a human lung using one to three static images to compare regional ventilation and pressure drop. Ibrahim et al. (2016) studied nonlinear deformation of the rat central airways using dynamic images. Therefore, the objective of this study is to investigate the effects of rigid vs. deforming airways, linear vs. nonlinear deformations, and static vs. dynamic imaging on particle transport in the human central airways 
with a CFD with airway geometric models derived from different numbers of dynamic or static images.

\section{Material and methods}

\subsection{Study cases}

In this study we used a four-dimensional dynamic computed tomography (4DCT) and static CT images of the healthy 58-year-old male subject (Subject 6) acquired by Jahani et al. (2015). The 4DCT images consist of volumetric images at 13 time points during a breathing cycle with period of $4.34 \mathrm{sec}$, and the static CT images were acquired at functional residual capacity (FRC) and total lung capacity (TLC). Figures 1 (a)(b) show the lung volume $V_{L}$ and total flow rate $Q_{T}$ as a function of time $t$. The flow rate, trachea hydraulic diameter, and corresponding Reynolds number at peak inspiration (PI) were $50.0 \mathrm{~L} / \mathrm{min}, 18.9 \mathrm{~mm}$, and 3740, respectively. Details of demographic information and image acquisition methods were described by Jahani et al. (2015). The maximum generation number of the airways considered in this study was eight (Figure 1(c)).

In the default case we used dynamic images at 13 time points and a CFD mesh that consisted of 5.3 million tetrahedral elements with the average distance from the wall to the first grid 7.5 in wall unit at PI. In addition to the default case, we considered two cases (coarse and fine) for mesh sensitivity tests and four more cases (linear dynamic, LD; rigid dynamic, RD; linear static, LS; and rigid static, RS) to investigate the effects of rigid vs. deforming airways, linear vs. nonlinear deformations, and step-wise static vs. dynamic imaging on particle transport (Table 1). The models in linear (LD and LS) cases were derived from the images at end expiration (EE) and end inspiration (EI), while those in rigid (RD and RS) cases were based on the image at EI. The dynamic and static images were used in the dynamic (LD and RD) and 
static (LS and RS) cases, respectively. The static images at EE and EI were obtained by linear interpolation between FRC and TLC with respect to lung volume (Figure 2). The default vs. LD, LD vs. RD, and LD vs. LS would illustrate the effects of linear vs. nonlinear deformations, rigid vs. deforming airways, and static vs. dynamic imaging, respectively. We also considered the RS case because (a) most of the previous studies used one static image and (b) dynamic images or multiple static images are not always available.

\subsection{Air flow simulation}

We simulated air flow in deforming airway models with large eddy simulation model and arbitrary Lagrangian-Eulerian method to account for turbulent flow during inspiration due to the jet created at the glottal constriction:

$$
\begin{aligned}
& \nabla \cdot \mathbf{u}=\mathbf{0} \\
& \frac{\partial \mathbf{u}}{\partial t}+(\mathbf{c} \cdot \nabla) \mathbf{u}=-\frac{1}{\rho_{f}} \nabla p+\nabla \cdot\left\{\left(v+v_{T}\right)\left[\nabla \mathbf{u}+(\nabla \mathbf{u})^{T}\right]\right\}
\end{aligned}
$$

where $\mathbf{u}=$ fluid velocity, $\mathbf{c}=\mathbf{u}-\hat{\mathbf{u}}=$ convective velocity, $\hat{\mathbf{u}}=$ mesh velocity computed by means of a computational solid mechanics-based algorithm (Stein et al., 2003), $\rho_{f}=$ fluid density (1.2 $\left.\mathrm{kg} / \mathrm{m}^{3}\right), v=$ fluid kinematic viscosity $\left(1.5 \times 10^{-5} \mathrm{~m}^{2} / \mathrm{s}\right)$, and $v_{T}=$ subgrid-scale eddy viscosity (Vreman, 2004). The verification and validation of our CFD model on turbulent channel flow and pulmonary air flow were presented by Lin et al. (2007) and Choi et al. (2010).

We imposed boundary conditions above the glottal constriction, at the distal end of our computational domain, and on airway wall using a synthetic eddy method (Jarrin et al., 2006), an image registration technique (Yin et al., 2013), and a volume-filling method (Tawhai, 2004). When the Reynolds number in the trachea was higher than 460 (Miyawaki et al., 2012), we 
introduced artificial turbulence that is correlated in space and time to the uniform velocity profile above the glottal constriction.

Image registration provided air volume in each voxel, which was connected to the distal ending branches via the skeleton generated by the volume-filling method, yielding regional volume $V_{R}$ that each ending branch ventilates. The flow rate in each ending branch $Q_{E}$ is the first derivative of $V_{R}$ with respect to time:

$Q_{E}=\frac{d V_{R}}{d t}=\frac{d V_{R}}{d V_{L}} \frac{d V_{L}}{d t}=\frac{d V_{R}}{d V_{L}} Q_{T}$

Because $V_{R}\left(V_{L}\right)$ was linear for most of ending branches, $d V_{R} / d V_{L}$ was a constant value for each branch. $d V_{R} / d V_{L}$ was computed with $V_{R}$ and $V_{L}$ at EE and EI for all cases except for the RS case, for which the fraction of $V_{R}$ at TLC was used instead.

Image registration also provided displacement fields in the lung, which was used to impose displacement boundary condition on airway wall. As displacement $\Delta \mathbf{x}$ was interpolated with respect to $V_{L}$, i.e., $\Delta \mathbf{x}\left(V_{L}\right)$, during breathing, airway deformation $\mathbf{x}(t)=\mathbf{x}\left(V_{L}(t)\right)$ was nonlinear even when using two images in the LD and LS cases.

\subsection{Particle simulation}

We computed particle trajectories with the Lagrangian tracking algorithm as a post process of air flow simulation:

$$
\frac{d \mathbf{u}_{p}}{d t}=\mathbf{f}_{D}+\frac{\rho_{p}-\rho_{f}}{\rho_{p}} \mathbf{g}
$$


where $\mathbf{u}_{p}=$ particle velocity, $\mathbf{f}_{D}=$ drag force per unit mass (Morsi and Alexander, 1972), $\rho_{p}=$ particle density, and $\mathbf{g}=$ gravitational acceleration. Our particle model on pulmonary particle transport was validated in our previous study (Miyawaki et al., 2012). Spherical particles with aerodynamic diameter $d_{a e}$ of $1.0,2.5,5.0,10$, and $20 \mu \mathrm{m}$ were considered, because we were interested in particle transport in human central airways. At the beginning of the breathing, i.e., EE, 10,000 particles were released uniformly in a cross-section above the glottal constriction. The particles were considered to be deposited when the distance between the particle center and airway wall was less than the particle radius. We only considered particle transport during inspiration as we did not simulate particle transport in the region that was not resolved by imaging.

\subsection{Analysis}

Particle distribution was quantified using three variables: distribution ratio $D R$, deposition efficiency $D E$, and deposition fraction $D F$ :

$$
\begin{aligned}
& D R=\frac{N_{p, e n t}}{M_{p, e x i t}} \\
& D E=\frac{N_{p, e n t}-N_{p, e x i t}}{N_{p, e n t}} \\
& D F=\frac{N_{p, e n t}-N_{p, e x i t}}{N_{p}}
\end{aligned}
$$

where $M_{p, e x i t}=$ number of particles exiting the parent branch of the region of interest, $N_{p, e n t}=$ number of particles entering the region of interest, $N_{p \text {,exit }}=$ number of particles exiting the region 
of interest, $N_{p}=$ total number of particles, and the region of interest is lung, lobe, or branch. Similar to $D R$, flow rate ratio $F R$ is defined as

$$
F R=\frac{Q}{Q_{P}}
$$

where $Q$ is the flow rate in the branch of interest, and $Q_{P}$ is the flow rate in the parent branch. $D R, D E$, and $F R$ are local variables, while $D F$ is affected by other part of the lung. When the

region of interest was branch, we computed average $\mu$, average absolute difference $E$, and average normalized difference $\varepsilon$ :

$$
\begin{aligned}
& \mu_{\phi}=\frac{1}{N_{b}} \sum_{i=1}^{N_{b}} \phi_{i} \\
& E_{\phi}=\frac{1}{N_{b}} \sum_{i=1}^{N_{b}}\left|\phi_{i}-\phi_{0, i}\right| \\
& \varepsilon_{\phi}=\frac{1}{N_{b}} \sum_{i=1}^{N_{b}} \frac{\left|\phi_{i}-\phi_{0, i}\right|}{\phi_{0, i}}
\end{aligned}
$$

where $\phi$ is a variable for a case, $N_{b}$ can be the number of all branches or number of branches at each generation, and $\phi_{0}$ is a variable for the default case.

\section{Results}

3.1 Mesh and particle sizes

In order to quantify the sensitivity of particle transport to grid size of the CFD mesh (Table 1), we computed $D R$ and $D E$ of 1.0 - to $20-\mu \mathrm{m}$ particles for each branch for the coarse, default (medium), and fine cases, and averaged the absolute difference between cases over branches 
(Figures 3(a)(b)). The differences between the default vs. fine cases were smaller than that between the default vs. coarse cases for both $D R$ and $D E$, and the former was overall $56 \%$ of the latter for $D R$ and $61 \%$ for $D E$. Therefore, we adopted the mesh for the default case in the following analysis, and the difference between the default vs. fine cases was considered as the uncertainty when comparing the default case with other cases.

In this study we only considered human central airways. Large particles may not reach all the central airways considered, and particle transport cannot be evaluated in those branches. On the other hand, small particles may not deposit and leave the central airways, resulting in a particle deposition smaller than the uncertainty of the model (Miyawaki et al., 2012). To find the optimal particle size that would cause well-distributed particle deposition over the central airways for the following analyses, we computed $D F$ for each branch and averaged it over branches at each generation (Figure 3(c)). A large portion of $20-\mu \mathrm{m}$ particles deposited at the first-generation airways compared to others, while a rather small fraction of $5.0-\mu \mathrm{m}$ particles deposited overall. Therefore, we considered the particles with $d_{a e}$ of $10 \mu \mathrm{m}$ in the following analyses.

\subsection{Global difference}

To compare the overall particle deposition in the LD, RD, LS, and RS cases with the default case, we computed $Q, D R$, and $D E$ for each branch and averaged absolute differences between the default and other cases over branches (Figure 4). Because $Q$ obviously depends on generation number, absolute difference for $Q$ was normalized by the value for the default case branch by branch before averaging. The average normalized absolute difference $\varepsilon$ in $Q\left(\varepsilon_{Q}\right)$ were zero in the dynamic cases, more than $10 \%$ for the LS case, and more than $20 \%$ for the RS case. The average absolute differences $E$ in $D R$ and $D E\left(E_{D R}\right.$ and $\left.E_{D E}\right)$ for the LD case (effect of nonlinear 
deformation) were smaller than the uncertainty, while $E_{D R}$ and $E_{D E}$ for the other cases were larger. More specifically, $E_{D R}$ and $E_{D E}$ for the RD case (effect of deformation) were 1-2\% points larger than the uncertainty, and those for the static (LS and RS) cases (effect of static imaging) were more than twice (5\% points) larger.

\subsection{Serial distribution}

To compare the serial particle distributions over generations in different cases, we computed $D F$ for each branch and averaged it over branches at each generation (Figure 5). Because sixthgeneration branches were not well-distributed in the lung, we only considered up to the fifthgeneration branches. Regarding incremental distribution $\mu_{\mathrm{DF}}$ (Figure 5(a)), similar to global particle deposition, only the LD case was within the uncertainty. The bias from the default case was not consistent over generations, while the RD, LS, and RS cases showed consistent bias from the default case at each generation. The bias of the three cases from the default case was particularly large at the second, third, and fifth generations. For cumulative distribution (Figure 5(b)), in addition to the LD case, the RS case was overall within the uncertainty because the difference in incremental distribution cancelled out. In contrast, the RD and LS cases overall underestimated the deposition.

\subsection{Parallel distribution}

To compare the parallel particle distributions over lungs and lobes in different cases, we computed $F R$ and $D R$ for lungs and lobes. $D E$ was not computed because it depends on the number of branches resolved by imaging. For example, if more branches were resolved in the upper lobes than in the lower lobes, $D E$ for the former would be probably larger. Compared to the dynamic (default, LD, and RD) cases, more air ventilated to the left lung in the static (LS and 
RS) cases due to the difference in images (Figure 6(a)). DR was within the uncertainty for all cases except the RS case, for which slightly ( $0.3 \%$ points) more particles entered the left lung compared to the uncertainty (Figure 6(b)).

Regarding lobar distribution, more air ventilated to the left upper lobe (LUL), right upper lobe (RUL), and right middle lobe (RML) for the static cases compared to the dynamic cases, and the difference from the dynamic cases was larger for the RS case because the regional ventilation was determined by one volumetric image (Figure 7(a)). Slightly ( 1\% points for LUL and $\sim 2 \%$ points for RUL) more particles entered the LUL and RUL for the static cases, and more particles entered the RML for the RD cases in addition to the static cases. The difference from the default case was smallest for the RD case $(+7.5 \%$ points) and largest for RS case $(+28.5 \%$ points) (Figure $7(b))$.

\subsection{Particle density}

Because the difference in $D R$ for the RML (and RLL) between the cases was the largest, we computed the cumulative density of particles $n_{p}$ that passed cross-sections at the distal end of right intermediate bronchus (BronInt), where airway bifurcates to enter RML and RLL, and more proximal cross-sections (the right main bronchus, $\mathrm{RMB}$, and trachea) during inspiration to visualize more detailed particle distribution (Figure 8). For the RD, LS, and RS cases, high particle density region was shifted to the RML side of the cross-section at the distal end of BronInt, while it was located near the center of the cross-section for the default case (only the default and LS cases are shown in Figure 8). In addition, the high particle density region already appeared to the RML side of the cross-section at the distal end of RMB for the RD, LS, and RS cases, while particles were well distributed to the BronInt (RML+RLL) side for the default case. 


\subsection{Airway motion}

Airway motion was quantified with airway wall velocity $U_{\text {wall }}$ normalized by air bulk velocity $U$ at peak inspiration for the default case (Figure 9). $U_{\text {wall }}$ was overall $0.33 \%$ of $U$ in the branches with generation number of 1 to 5 , and mean and standard deviation of $U_{\text {wall }} / U$ increased with generation number. Mean + standard deviation of $U_{\text {wall }} / U$ was less than $1.0 \%$ at the 5 th generation.

\section{Discussion}

We have sought to assess the effect of rigid vs. deforming airways, linear vs. nonlinear airway deformations, and static vs. dynamic imaging on estimates of particle transport in human central airways. We simulated particle motion with our previously-validated 3-D CFD-based computer lung model for the default, LD, RD, LS, and RS cases, for which different numbers of dynamic or static CT-images were used (Table 1). In particular, we compared global particle distribution, global particle deposition, serial particle deposition (over generations), and parallel particle distribution (over lungs and lobes) between the above five cases.

As expected, the global differences in particle distribution and deposition (combination of $D R$ and $D E$ ) compared to the default case (13 time points) were larger for the two-time-point static (LS) case than the two-time-point dynamic (LD) case because the volumetric configurations (airway and parenchyma) at the two end points are quite different for statically vs. dynamically acquired images. Considering the uncertainty due to mesh size, the difference for the LD case was negligible, while that for the LS case was about $7 \%$. This implies that the global effect of static vs. dynamic imaging on particle distribution and deposition is about $7 \%$. On the other hand, the difference for the RD case was about $2 \%$ more than the uncertainty, so the effect 
of rigid vs. deforming airways is about $2 \%$. Therefore, the difference in regional ventilation may contribute to the effect of static vs. dynamic imaging more than difference in airway geometry does. If only one static image is available, regional ventilation would be less accurate than that based on two static images even with the aid of air volume information at TLC (Miyawaki et al., 2015). Based on the results of this study, however, particle deposition and distribution does not seem to be sensitive to this difference at global level.

Serial distribution of particle deposition is important when considering the relationship between function and structure of central airways because structure of airways in asthmatic and smokers are different from healthy subjects even for central airways (Montaudon et al., 2009a, 2009b). Effect of rigid vs. deforming airways on serial distribution of particle deposition (the LD vs. RD cases) can be compared to the results for the moving mesh and oscillating flow cases reported by Mead-Hunter et al. (2013). In our study, average deposition fraction for the RD case underestimated or overestimated that for the LD case by $22 \%$ on average, depending on generation number, while the cumulative average deposition fraction for the RD case consistently underestimated and the difference was $13 \%$ at the fourth generation. In contrast, deposition fraction for the oscillating flow (rigid airway with transient flow rate) case consistently overestimated the moving mesh case by about $1,100 \%$ on average, and cumulative deposition fraction was also overestimated to cause $430 \%$ difference at the fourth generation in Mead-Hunter et al. (2013).

This difference could be attributed to the difference in simulation conditions. In MeadHunter et al. (2013), particle size and flow rate were much smaller than the present study, resulting in a Stokes number smaller by a factor of 250 (Table 2). In fact, cumulative deposition fraction at the fourth generation was smaller by a factor of 7 . In addition, airway wall velocity 
was $0.33( \pm 0.17) \%$ of air bulk velocity in 4 th-generation branches in the present study, while it was about $100 \%$ in the moving mesh case by Mead-Hunter et al. (2013) because net air flow across the distal end of 4th-generation branches was zero. Furthermore, geometry for the rigid airway case was based on EI in the present study, causing lower velocity and less particle deposition, while it was based on EE in Mead-Hunter et al. (2013), resulting in higher velocity and more deposition.

The difference in particle distribution was within the uncertainty for lungs but not for lobes. For example, $18 \%$ more particles entered the RML for the static (LS) case than the dynamic (LD) case, so static vs. dynamic imaging may affect the particle distribution between RML and RLL by $18 \%$. This may be large enough to affect, e.g., heterogeneity of aerosol deposition for lobes (Greenblat et al., 2015). Because more (2.5\%) air entered the RML for the static case, the difference in particle distribution could be caused by the difference in flow rate distribution. The flow rate distribution at proximal branching points could also contribute to the difference in particle distribution. It is possible that particles were not well-mixed and remained to the RML side of RMB and BronInt with weaker secondary flow after the first bifurcation because smaller amount of air entered the RMB for the LS case.

Limitations of this study and potential improvements include the fact that this is a study utilizing a single healthy subject. When including the effects of diseases such as asthma and chronic obstructive lung disease, static vs. dynamic effects are likely to be much bigger. In addition, the extrathoracic region was modeled with a physiologically-consistent laryngeal model instead of a CT-based model, but the air flow field and particle distribution predicted by the former was consistent with that predicted by the latter (Miyawaki et al., 2013). Furthermore, since the uncertainty in this study was defined as the difference between the default (medium) vs. 
fine cases, it could vary when a finer mesh is used. This limitation could possibly change some results. For example, with the current mesh the difference between the default vs. linear dynamic (LD) cases was smaller than the uncertainty, but with a finer mesh it could become larger than the uncertainty.

\section{Conclusions}

This study demonstrated that the difference in regional flow rate, as opposed to airway geometry, between static vs. dynamic imaging primarily contributed to the difference in particle distribution and deposition in the human central airways. Serial particle distribution over generations estimated with rigid airways was shown to be significantly different from that with deforming airways, but the difference was significantly smaller than reported in a previous study. Regarding parallel particle distribution over lobes, the effect of static vs. dynamic imaging was larger than the effect of rigid vs. deforming airways. The results presented here provide quantitative uncertainty in estimating particle distribution and deposition with rigid or deforming airway models derived from static imaging for the development of pulmonary pathology and aerosolized pharmaceutical drugs.

\section{Acknowledgements}

This work was supported in part by NIH grants R01-HL094315, U01-HL114494, R01HL112986, S10-RR024738, and S10-RR022421. We would like to thank Nariman Jahani for his help with utilization of the 4D CT data and associated physiologic parameters. We also thank the Extreme Science and engineering Discovery Environment (XSEDE) (allocation MCA07S015) sponsored by the National Science Foundation that provided the computational time at San Diego Supercomputer Center (SDSC) and the Texas Advanced Computing Center (TACC). 


\section{Disclosures}

Eric A. Hoffman is a founder and shareholder of VIDA Diagnostics, a company commercializing lung image analysis software developed, in part, at the University of Iowa.

\section{References}

Choi, J., Tawhai, M. H., Hoffman, E. A., \& Lin, C. L. (2009). On intra-and intersubject variabilities of airflow in the human lungs. Physics of Fluids, 21, 101901.

Choi, J., Xia, G., Tawhai, M. H., Hoffman, E. A., \& Lin, C.-L. (2010). Numerical study of highfrequency oscillatory air flow and convective mixing in a CT-based human airway model. Annals of biomedical engineering, 38, 3550-3571.

Darquenne, C., Fleming, J. S., Katz, I., Martin, A. R., Schroeter, J., Usmani, O. S., Venegas, J., \& Schmid, O. (2016). Bridging the Gap Between Science and Clinical Efficacy: Physiology, Imaging, and Modeling of Aerosols in the Lung. Journal of Aerosol Medicine and Pulmonary Drug Delivery, (in press).

De Backer, J. W., Vos, W. G., Vinchurkar, S. C., Claes, R., Drollmann, A., Wulfrank, D., Parizel, P. M., Germonpré, P., \& De Backer, W. (2010). Validation of Computational Fluid Dynamics in CT-based Airway Models with SPECT/CT. Radiology, 257, 854-862.

Dubsky, S. \& Fouras, A. (2015). Imaging regional lung function: A critical tool for developing inhaled antimicrobial therapies. Advanced Drug Delivery Reviews, 85, 100-109.

Greenblatt, E. E., Winkler, T., Harris, R. S., Kelly, V. J., Kone, M., Katz, I., Martin, A. R., Caillibotte, G., \& Venegas, J. (2016). What Causes Uneven Aerosol Deposition in the Bronchoconstricted Lung? A Quantitative Imaging Study. Journal of Aerosol Medicine and Pulmonary Drug Delivery, 29, 57-75.

Grgic, B., Finlay, W. H., Burnell, P. K. P., \& Heenan, A. F. (2004). In vitro intersubject and 
intrasubject deposition measurements in realistic mouth-throat geometries. Journal of Aerosol Science, 35, 1025-1040.

Huang, J., Sun, H., Liu, C., \& Zhang, L. (2013). Moving boundary simulation of airflow and micro-particle deposition in the human extra-thoracic airway under steady inspiration. Part I: Airflow. European Journal of Mechanics - B/Fluids, 37, 29-41.

Ibrahim, G., Rona, A., \& Hainsworth, S. (2015). Non-uniform central airways ventilation model based on vascular segmentation. Computers in Biology and Medicine, 65, 137-145.

Ibrahim, G., Rona, A., \& Hainsworth, S. V. (2016). Modeling the Nonlinear Motion of the Rat Central Airways. Journal of Biomechanical Engineering, 138, 011007-011007.

Jahani, N., Choi, S., Choi, J., Iyer, K., Hoffman, E. A., \& Lin, C.-L. (2015). Assessment of regional ventilation and deformation using 4D-CT imaging for healthy human lungs during tidal breathing. Journal of Applied Physiology, 119, 1064-1074.

Jarrin, N., Benhamadouche, S., Laurence, D., \& Prosser, R. (2006). A synthetic-eddy-method for generating inflow conditions for large-eddy simulations. International Journal of Heat and Fluid Flow, 27, 585-593.

Lin, C. L., Tawhai, M. H., McLennan, G., \& Hoffman, E. A. (2007). Characteristics of the turbulent laryngeal jet and its effect on airflow in the human intra-thoracic airways. Respiratory physiology \& neurobiology, 157, 295-309.

Ma, B. \& Lutchen, K. R. (2009). CFD simulation of aerosol deposition in an anatomically based human large-medium airway model. Annals of Biomedical Engineering, 37, 271-285.

Mead-Hunter, R., King, A. J., Larcombe, A. N., \& Mullins, B. J. (2013). The influence of moving walls on respiratory aerosol deposition modelling. Journal of Aerosol Science, 64, 48-59.

Miyawaki, S., Hoffman, E. A., \& Lin, C.-L. (2015). CFD simulations of a deforming human lung 
using dynamic and static CT images. Bulletin of the American Physical Society, 60.

Miyawaki, S., Tawhai, M. H., Hoffman, E. A., \& Lin, C.-L. (2013). An image-based automatic mesh generation and numerical simulation for a population-based analysis of aerosol delivery in the human lungs. Bulletin of the American Physical Society, 58.

Miyawaki, S., Tawhai, M. H., Hoffman, E. A., \& Lin, C.-L. (2012). Effect of carrier gas properties on aerosol distribution in a CT-based human airway numerical model. Annals of biomedical engineering, 40, 1495-1507.

Montaudon, M., Berger, P., Lederlin, M., Marthan, R., de Lara, J. M. T., \& Laurent, F. (2009). Bronchial morphometry in smokers: comparison with healthy subjects by using 3D CT. European radiology, 19, 1328-1334.

Montaudon, M., Lederlin, M., Reich, S., Begueret, H., de Lara, J. M. T., Marthan, R., Berger, P., \& Laurent, F. (2009). Bronchial Measurements in Patients with Asthma: Comparison of Quantitative Thin-Section CT Findings with Those in Healthy Subjects and Correlation with Pathologic Findings1. Radiology, 253, 844-853.

Morsi, S. A. \& Alexander, A. J. (1972). An investigation of particle trajectories in two-phase flow systems. Journal of Fluid Mechanics, 55, 193-208.

Stein, K., Tezduyar, T., \& Benney, R. (2003). Mesh moving techniques for fluid-structure interactions with large displacements. Journal of Applied Mechanics, 70, 58.

Vreman, A. W. (2004). An eddy-viscosity subgrid-scale model for turbulent shear flow: Algebraic theory and applications. Physics of Fluids, 16, 3670.

Yin, Y., Choi, J., Hoffman, E. A., Tawhai, M. H., \& Lin, C. L. (2010). Simulation of pulmonary air flow with a subject-specific boundary condition. Journal of Biomechanics, 43, 21592163.

Yin, Y., Choi, J., Hoffman, E. A., Tawhai, M. H., \& Lin, C.-L. (2013). A multiscale MDCT 
image-based breathing lung model with time-varying regional ventilation. Journal of Computational Physics, 244, 168-192. 
Table 1 . Summary of study cases. $\mathrm{LD}=$ linear dynamic, $\mathrm{RD}=$ rigid dynamic, $\mathrm{LS}=$ linear static, $\mathrm{RS}=$ rigid static, $\mathrm{EE}=$ end expiration, $\mathrm{EI}=$ end inspiration, and the grid size is the average distance from the wall to the first grid at peak inspiration in wall unit. The number of tetrahedral elements for coarse, default, and fine cases were 1.5, 5.4, and 11.7 million, respectively.

\begin{tabular}{cccccccc}
\hline Case & 1 & 2 & 3 & 4 & 5 & 6 & 7 \\
\hline Name & default & coarse & fine & LD & RD & LS & RS \\
Imaging & 4 DCT & 4 DCT & 4 DCT & 4DCT & 4DCT & CT & CT \\
Images / cycle & 13 & 13 & 13 & EE, EI & EI & EE, EI & EI \\
Grid size & 7.5 & 11.4 & 5.7 & 7.5 & 7.5 & 7.5 & 7.5 \\
\hline
\end{tabular}


Table 2. Difference in simulation conditions in the trachea at peak inspiration (PI) between the present study and Mead-Hunter et al. (2013). It was assumed that the air dynamic viscosity was $1.8 \times 10^{-5} \mathrm{~kg} / \mathrm{m} \cdot \mathrm{s}$ and particle density was $1,000 \mathrm{~kg} / \mathrm{m}^{3}$ in Mead-Hunter et al. (2013).

\begin{tabular}{ccc}
\hline & Present & $\begin{array}{c}\text { Mead-Hunter } \\
\text { et al. (2013) }\end{array}$ \\
\hline Particle aerodynamic & 10 & 1.0 \\
diameter $(\mu \mathrm{m})$ & 50 & 12 \\
Flow rate $(\mathrm{L} / \mathrm{min})$ & 19 & 16 \\
Diameter $(\mathrm{mm})$ & $4.77 \times 10^{-2}$ & $1.92 \times 10^{-4}$ \\
Stokes number & & \\
\hline
\end{tabular}


Figure 1. (a) Lung volume $V_{L}$ and (b) total flow rate $Q_{T}$ as a function of time $t$. (c) Airway wall geometry at end expiration EE (3.39 L) and end inspiration EI (4.37 L). FRC = functional residual capacity $(2.51 \mathrm{~L}), \mathrm{TLC}=$ total lung capacity $(6.36 \mathrm{~L}), \mathrm{PI}=$ peak inspiration $(50.0$ $\mathrm{L} / \mathrm{min}), \mathrm{PE}=$ peak expiration $(-51.8 \mathrm{~L} / \mathrm{min})$.

Figure 2. (a) Time $t$, (b) regional volume $V_{R}$ of lobes, and (c) z-coordinate of a point on airway wall as a function of lung volume $V_{L}$. The dashed lines in (b) denote static image, and the dashed line in (c) denotes expiration. $\mathrm{LUL}=$ left upper lobe, $\mathrm{LLL}=$ left lower lobe, $\mathrm{RUL}=$ right upper lobe, $\mathrm{RML}=$ right middle lobe, $\mathrm{RLL}=$ right lower lobe, default $=$ default case, $\mathrm{LD}=$ linear dynamic case, and LS = linear static case.

Figure 3. Average absolute differences in (a) distribution ratio $E_{D R}$ and (b) deposition efficiency $E_{D E}$ between the default vs. coarse cases and the default vs. fine cases. (c) Average deposition fraction $\mu_{D E}$ for 5.0-, 10-, and 20- $\mu \mathrm{m}$ particles for the default case. $d_{a e}=$ aerodynamic diameter.

Figure 4. (a) Average normalized absolute difference in flow rate $\varepsilon_{Q}$ and average absolute differences in (b) particle distribution ratio $E_{D R}$, and (c) particle deposition efficiency $E_{D E}$ for 10$\mu \mathrm{m}$ particles compared to the default case. The gray region indicates the default vs. fine cases. $\mathrm{LD}=$ linear dynamic, $\mathrm{RD}=$ rigid dynamic, $\mathrm{LS}=$ linear static, $\mathrm{RS}=$ rigid static.

Figure 5. (a) Average deposition fraction $\mu_{D F}$ and (b) cumulative average deposition fraction $\Sigma \mu_{D F}$ for $10-\mu \mathrm{m}$ particles as a function of generation number. The gray region indicates the difference between the default and fine cases. 
Figure 6. (a) Flow rate ratio $F R$ and (b) particle distribution ratio $D R$ for $10-\mu \mathrm{m}$ particles in the left and right lungs. The gray region indicates the difference between the default and fine cases. $F R_{\text {Left }}+F R_{\text {Right }}=D R_{\text {Left }}+D R_{\text {Right }}=100 \%$

Figure 7. (a) Flow rate ratio $F R$ and (b) particle distribution ratio $D R$ for $10-\mu \mathrm{m}$ particles in the left upper lobe (LUL), left lower lobe (LLL), right upper lobe (RUL), right middle lobe (RML), and right lower lobe (RLL). The gray region indicates the difference between the default and fine cases. $F R_{L U L}+F R_{L L L}=F R_{R M L}+F R_{R L L}=D R_{L U L}+D R_{L L L}=D R_{R M L}+D R_{R L L}=100 \%$.

Figure 8. Cumulative density of $10-\mu \mathrm{m}$ particles $n_{p}\left(/ \mathrm{mm}^{2}\right)$ that passed cross-sections at distal end of the trachea, right main bronchus, and right intermediate bronchus during inspiration for (a) the default and (b) LS cases. RUL = right upper lobe, $\mathrm{RML}=$ right middle lobe, $\mathrm{RLL}=$ right lower lobe.

Figure 9 . Average wall velocity $U_{\text {wall }}$ normalized by air bulk velocity $U$ at peak inspiration as a function of generation number for the default case. The error bars indicate standard deviation. 
Figure1
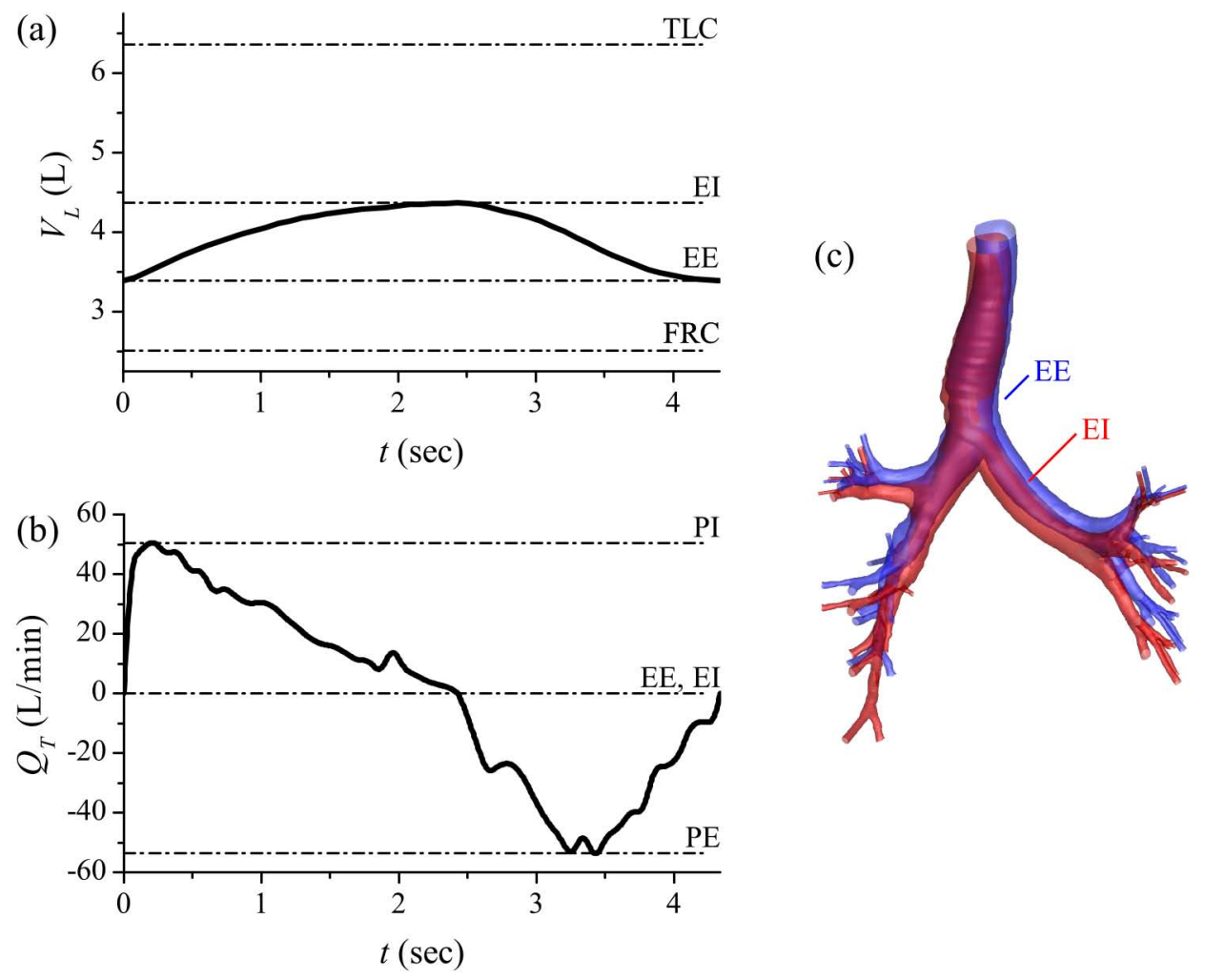
Figure2

(a)
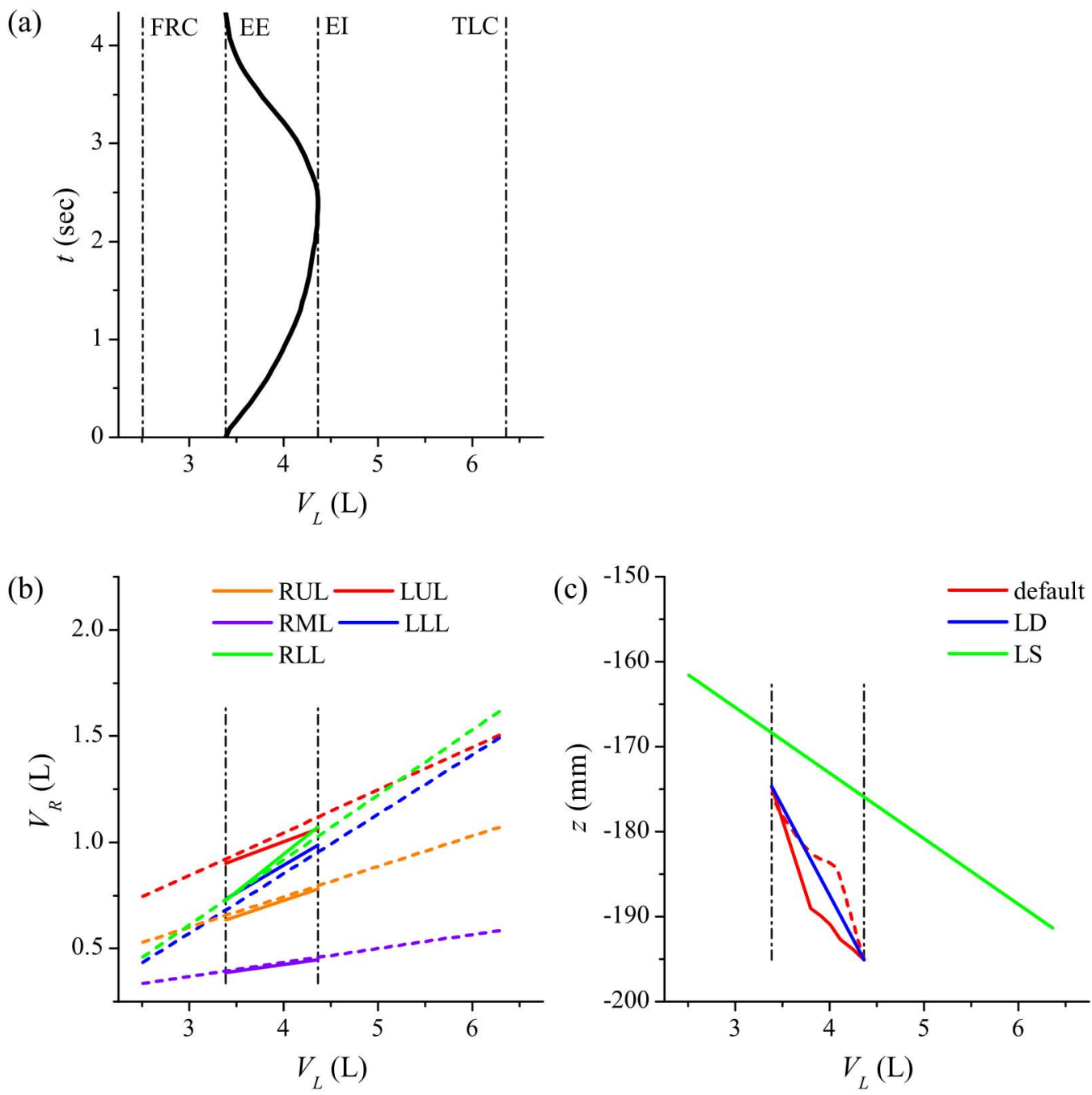


\section{Figure3}
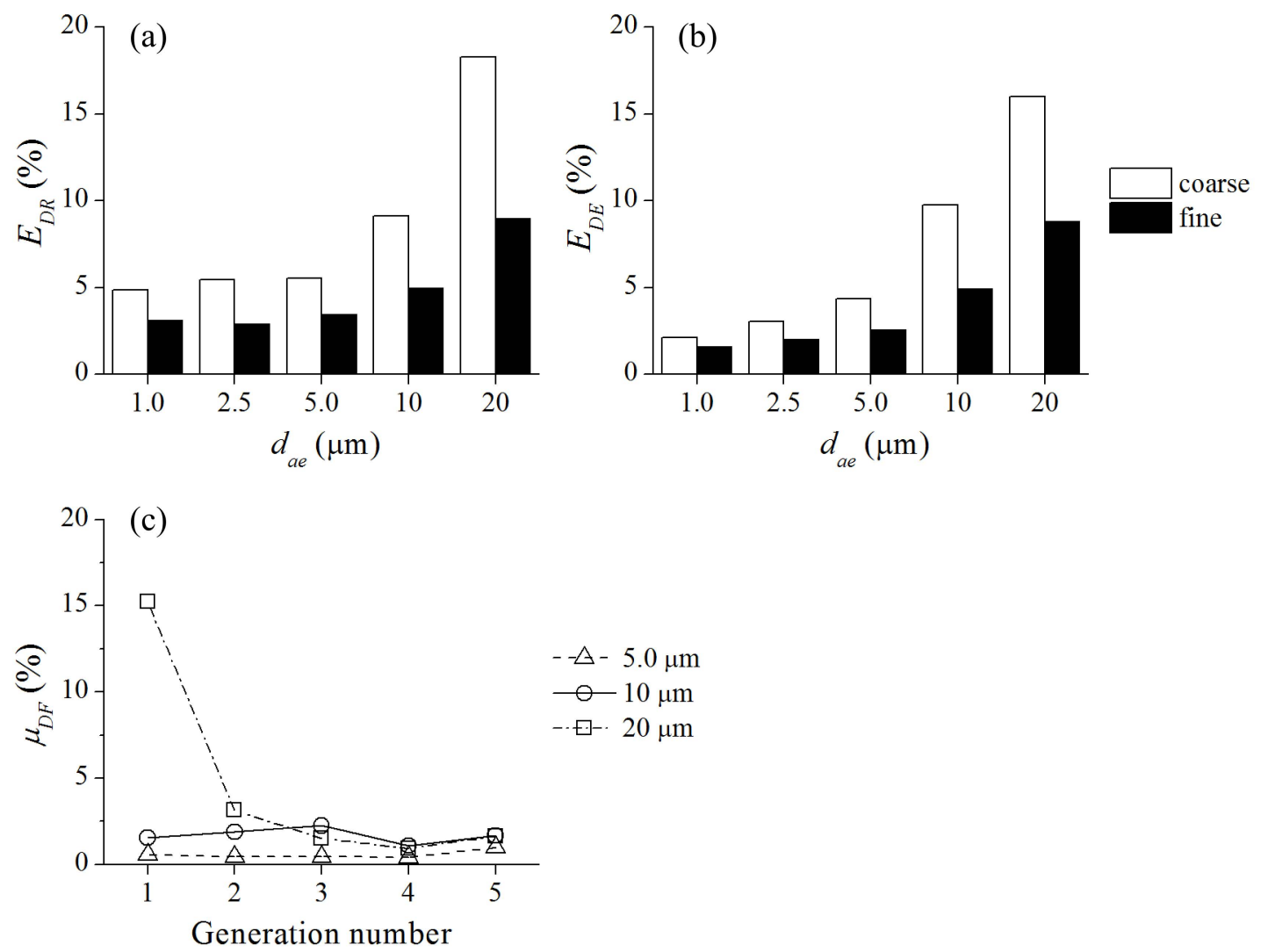
Figure4
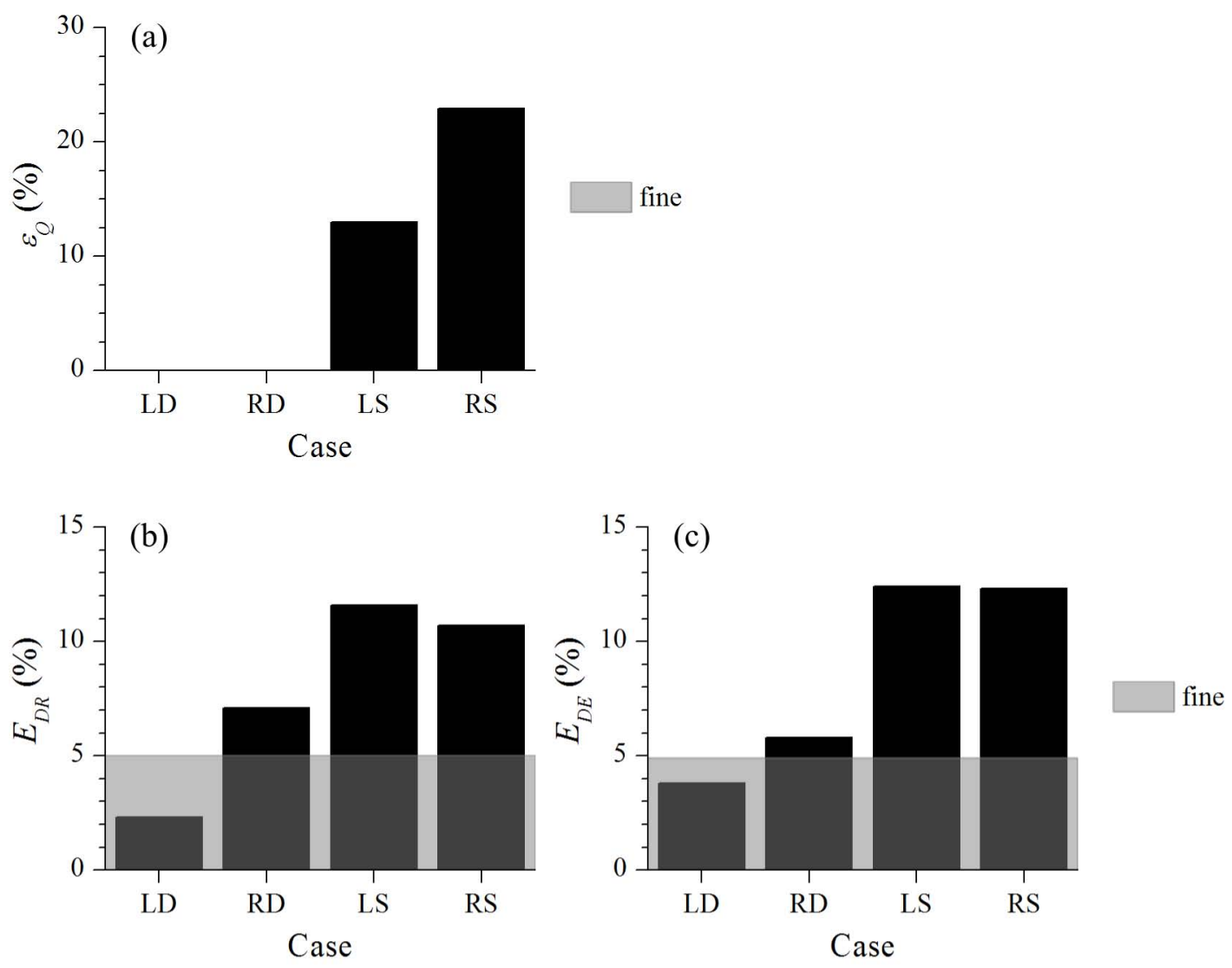


\section{Figure5}
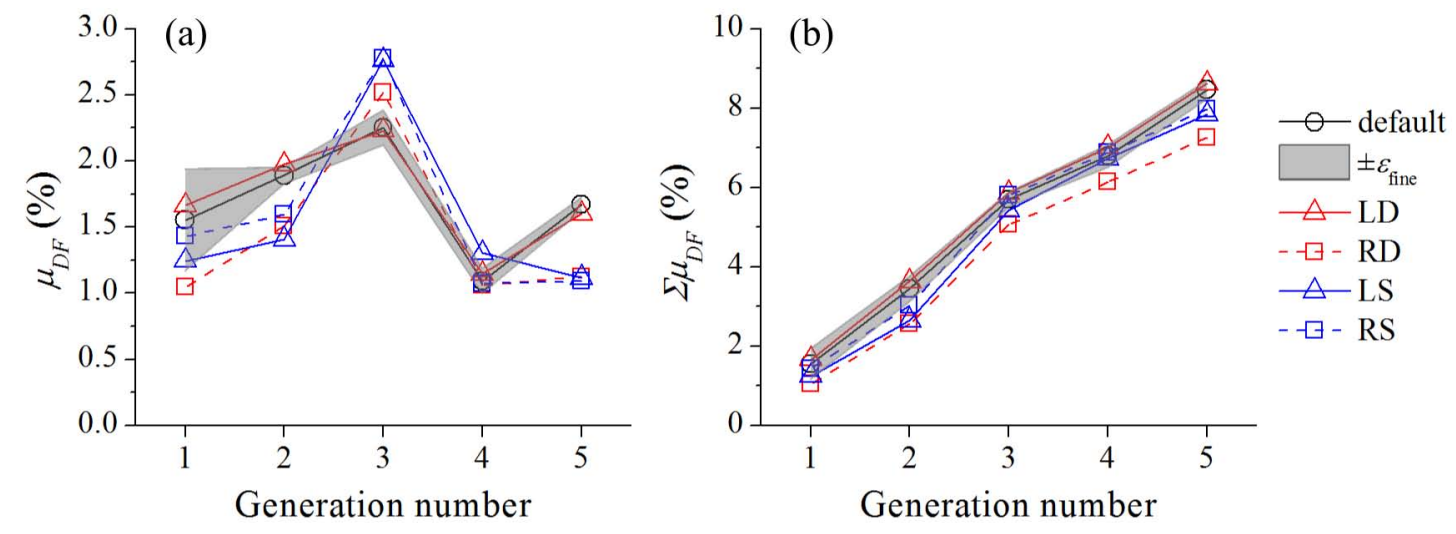
Figure6
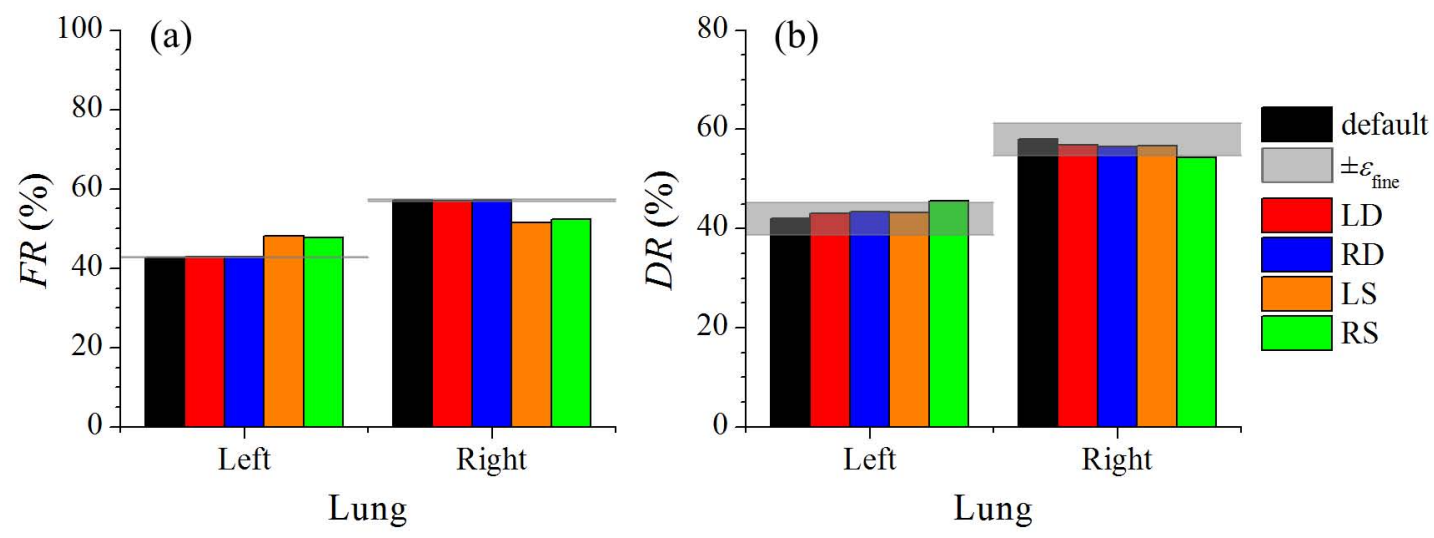
Figure7
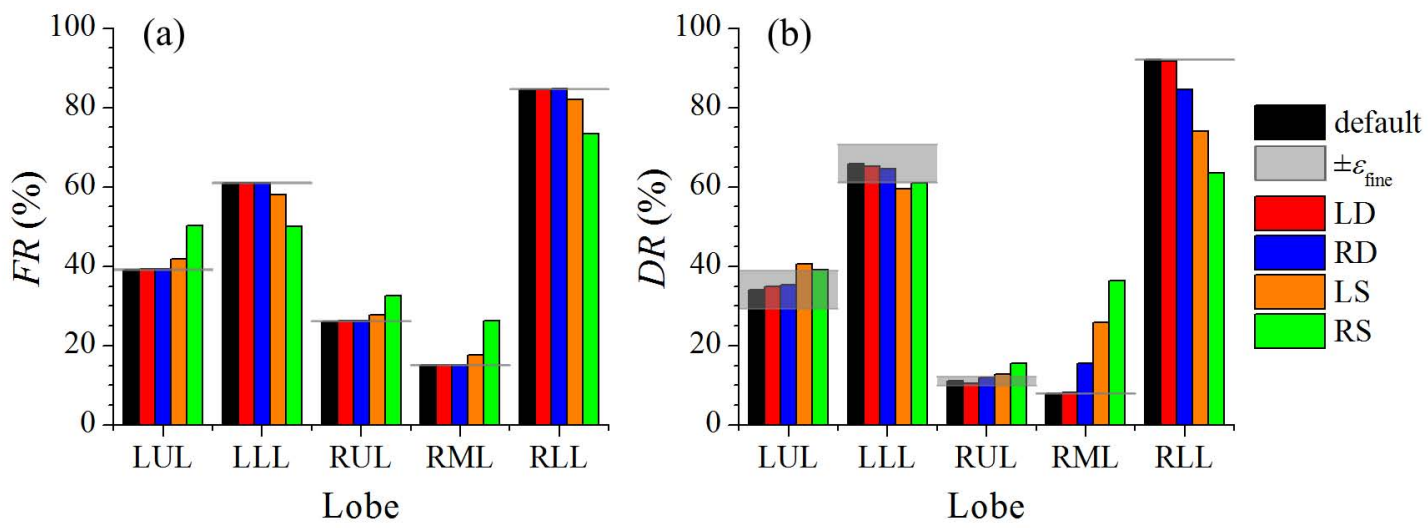
Figure8

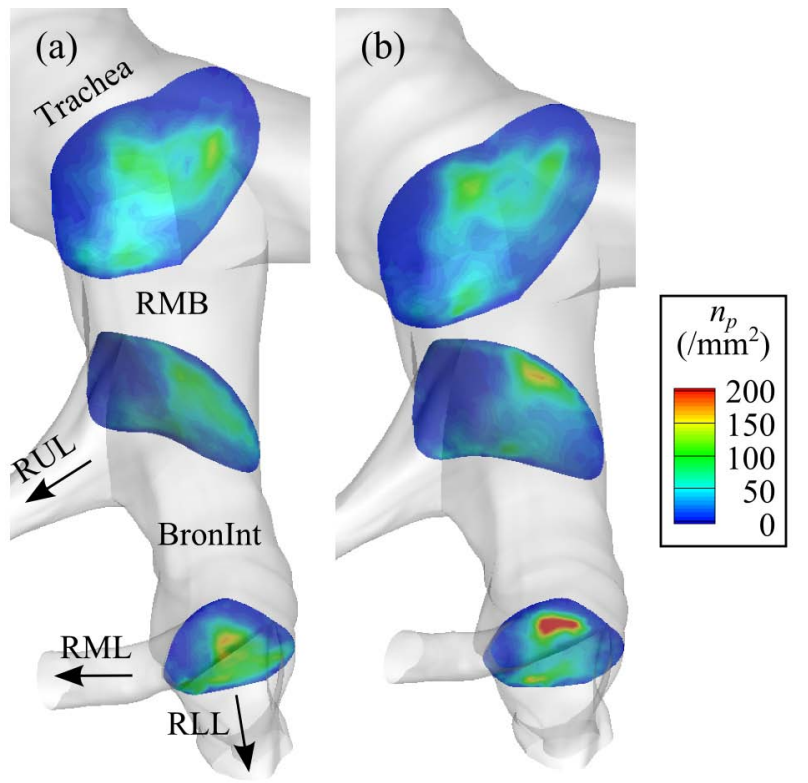


Figure9

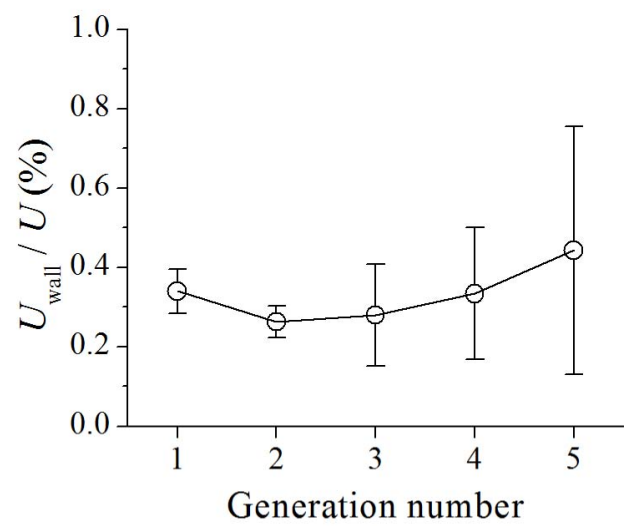

